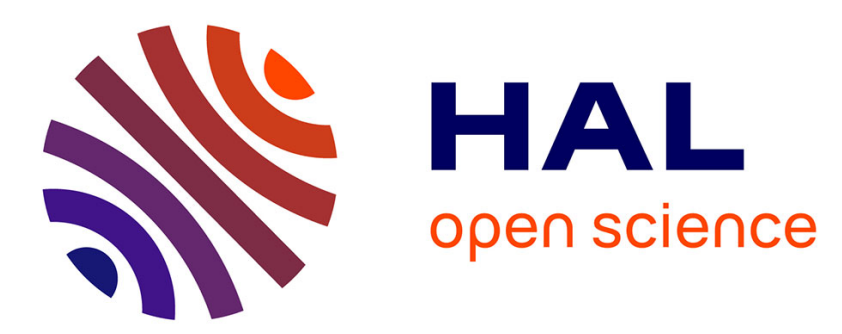

\title{
Services KPI-based Energy Management Strategies for Green Wireless Networks
}

\author{
Ali El Amine, Hussein Al Haj Hassan, Loutfi Nuaymi
}

\section{To cite this version:}

Ali El Amine, Hussein Al Haj Hassan, Loutfi Nuaymi. Services KPI-based Energy Management Strategies for Green Wireless Networks. 2018 IEEE 29th Annual International Symposium on Personal, Indoor and Mobile Radio Communications (PIMRC), Sep 2018, Bologna, Italy. pp.7, 10.1109/PIMRC.2018.8581023 . hal-01985902

\section{HAL Id: hal-01985902 https://imt-atlantique.hal.science/hal-01985902}

Submitted on 18 Jan 2019

HAL is a multi-disciplinary open access archive for the deposit and dissemination of scientific research documents, whether they are published or not. The documents may come from teaching and research institutions in France or abroad, or from public or private research centers.
L'archive ouverte pluridisciplinaire HAL, est destinée au dépôt et à la diffusion de documents scientifiques de niveau recherche, publiés ou non, émanant des établissements d'enseignement et de recherche français ou étrangers, des laboratoires publics ou privés. 


\title{
Services KPI-based Energy Management Strategies for Green Wireless Networks
}

\author{
Ali El-Amine *, Hussein Al Haj Hassan ${ }^{\dagger}$, Loutfi Nuaymi * \\ ${ }^{\star}$ Departement Système Réseaux, Cybersécurité et Droit du numérique (SRCD). IMT-Atlantique. Rennes, France \\ ${ }^{\dagger}$ Department of Computer and Communications Engineering. Faculty of Engineering. American University of \\ Science and Technology. Beirut, Lebanon \\ Email: `firstname.lastname@imt-atlantique.fr, †hhajhassan@aust.edu.lb
}

\begin{abstract}
Complex green wireless networks including smart grid, renewable energy and batteries need precise energy management strategies in order to realize some of the defined objectives. Although these networks have different services (streaming, web, voice call, etc.) with different requirements, most research work slightly covered the specific contribution of each of these services and their effect on the network energy consumption. In this paper, we consider advanced KPIs (Key Performance Indicators) putting forward each service contribution to energy consumption. Using these new KPIs, we propose to adapt some energy management strategies, leading to performance amelioration and new energy savings, under renewable energy and smart grid environment. Simulations indicate the efficiency of our proposed adaptation, achieving $11.45 \%$ enhancement in these new KPIs, and outperforming several benchmark algorithms with a gain up to $8 \%$ points in terms of energy savings reduction. We analyze the benefits of the services KPIs and also their limits, leading to possible new services-based efficient energy management strategies in the considered framework.
\end{abstract}

\section{INTRODUCTION}

The radical surge of Information and Communication Technology (ICT) devices and services has pushed corresponding energy consumption to grow at a staggering rate. Followed by an increased awareness of environmental protection and strict regulations on pollutant emissions, there is a growing attention to reduce the energy consumption associated with ICT equipment [1]. For instance, it is estimated that ICT consumes around $4.7 \%$ of the world's electrical energy, releasing into the atmosphere about $1.7 \%$ of the global $\mathrm{CO}_{2}$ emissions [2]

Cellular networks, a significant component of ICT energy consumption, have drawn considerable attention of many researchers from both academia and industry. With roughly $80 \%$ of network energy being consumed by base stations (BSs) [3], most efforts to improve energy efficiency (EE) in mobile radio networks focus on BSs.

\section{A. Related Work}

EE is becoming a significant key performance indicator (KPI) in evaluating next generation cellular networks. It is defined as the amount of information that can be reliably transmitted per Joule of consumed energy [4]. Due to its simplicity, this metric is widely used in the evaluation of the EE of telecommunication networks including cellular networks [5]. In the literature, recent efforts have been made investigating EE and energy savings of cellular networks. Among the different approaches existing in the literature, sleep mode strategies and the deployment of heterogeneous networks have been proven to be the most effective in improving energy savings and EE in cellular networks [6], [7]. Nevertheless, providing BSs with renewable energy (RE) sources is another effective approach to enhance the EE of cellular networks by acquiring clean and cheap energy from ambient surrounding. A detailed survey on powering cellular BSs with RE can be found in [8].

In order to benefit more from RE, intelligent energy management is required to yield a higher EE in cellular networks. In [9], we showed that intelligent usage of harvested energy results in higher cost savings in a variable electricity price environment. Based on state of charge (SoC) of the battery and electricity price, we introduced SPAEMA, an energy management algorithm, that decides whether to store or use RE. The work, however, is limited to one BS, and it does not study the impact on EE metric. In [10], the design of energy efficient heterogeneous cellular networks is investigated. Using a stochastic geometry-based model, the authors derived the EE with different sleep modes for small cells. However, the study was done in the absence of RE. In [11], maximizing the EE in a 2-tier wireless network powered by both RE and power grid is considered through power allocation. An offline algorithm based on parametric optimization is proposed. The work, however, ignores sleep mode strategies. In [12], EE in a multiuser energy harvesting system is considered. The authors developed an offline algorithm that maximizes the $\mathrm{EE}$ of the system by jointly varying the users' transmission time and power. Despite their analytical work, the model is limited to only one BS.

In most prior efforts, the emphasis was on maximizing cost savings or EE of the network. In [13], the authors assessed the sharing of energy consumption among the different service categories provided by the network. An energy sharing model based on Shapley value, is proposed and proven to be a good balance between the service categories, in terms of fairness compared to uniform and proportional sharing. To the best of our knowledge, the study of the impact of RE and energy management strategies on services KPIs has not been well investigated in the literature, and it is still an open problem. 


\section{B. Paper Contributions}

In this paper, we propose an EE metric that evaluates the performance of the network. In contrast to the definition given by ETSI [14], which limits the evaluation of the EE to operational BSs, we suggest an extension that includes inactive BSs (in sleep mode) and RE. Then, we assess this EE-KPI over all the services the network is providing by putting forward their contribution to energy consumption under RE sources. Finally, we propose to adapt some energy management techniques with the objective of having better energy indicators for each of these services

While most studies assume BSs fully equipped with RE sources, our model considers a more realistic scenario where a percentage of sites is equipped with energy harvesters. This renders the model economically viable when the network operator has thousands of deployed BSs.

The rest of the paper is outlined by the following sections: Section II describes the system model. Section III explains the different KPI metrics understudy and states the problem formulation. Then, we propose two algorithms to enhance this EE-KPI in Section IV. In Section $\mathrm{V}$, simulation results are presented and analyzed. Finally, Section VI concludes the discussion and future work.

\section{System Model}

\section{A. Network Description}

We consider a wireless cellular network overlaid with $M$ macro BSs having an inter-cell distance D and $K$ mobile users. We denote the set of BSs by $\mathcal{B}=\{1, \ldots, m, \ldots, M\}$, and the set of users served by BS $m$ by $\mathcal{U}_{m}=\left\{1, \ldots, u, \ldots, k_{m}\right\}$. Initially, the users are associated with and served by the BSs, based on best signal-to-interference-plus-noise ratio method (Best SINR) that is managed by a centralized energy management unit (EMU). Each user measures the SINRs by using pilot signals from all BSs and sends it to the EMU. These BSs are providing different services to the users (streaming, web, voice call, etc.). We further consider two sets of BSs. One is solely powered by conventional power grid, e.g. smart grid (SG), and is denoted by $B S^{\text {grid }}$. The other set of BSs is equipped with RE and energy storages (batteries); hence, it is powered by a mix of hybrid energy sources ( $\mathrm{SG}+\mathrm{RE})$ and is denoted by $B S^{m i x}$. The total bandwidth of the system $W$ is shared among the BSs with a frequency reuse factor of one. We consider that the traffic load, on-grid energy price and RE are all varying over time.

\section{B. Dynamic Network Operations}

As illustrated in Fig. 1, we divide our observation time $T$ into multiple one hour-periods, each with $L$ time slots. Each slot $s$ has a duration of $1 / L$ hour. At each slot, we allocate RE and set the state of each BS (active or sleep) following the energy management strategies explained below.

1) RE allocation: The decision of allocating RE is managed through a local EMU installed at each BS $\in B S^{m i x}$. Based on the current traffic load and SoC of the battery, the
EMU decides whether to use or store the harvested energy. The decisions of managing RE are detailed in Section IV.

2) BS sleep scheme: Based on the SINR measurements collected by all users, the centralized EMU controls the operational mode of the BSs (active or sleep) following our sleep mode strategy presented in [15]: SINR threshold-based method. This algorithm aggressively switches off candidate BSs $\left(\mathcal{B}^{c}\right)$ according to an SINR Switch-Off Threshold $\left(\mathrm{SINR}_{S O T}\right)$. While this algorithm considers only BSs $\in B S^{\text {grid }}$ to be switched off, we extend the set $\mathcal{B}^{c}$ to include BSs $\in B S^{\text {mix }}$. However, not all BSs $\in B S^{\text {mix }}$ are candidates to switch off. In Section IV-B, we detail the required conditions for a $\mathrm{BS} \in B S^{\text {mix }}$ to join $\mathcal{B}^{c}$. The motivation behind this selection is to give the BSs consuming energy solely from the grid the priority to switch off, in order to increase the utilization of RE.

A candidate BS $m$ switches off if the SINRs measured by the users served by BS $m$ from neighboring BSs $m^{\prime}$ are above the $\operatorname{SINR}_{S O T}$ :

$$
\begin{aligned}
& \operatorname{SINR}_{m^{\prime}}(u) \geq \operatorname{SINR}_{S O T}, \forall u \in \mathcal{U}_{m}, m \in \mathcal{B}^{c} \\
& \text { and } m^{\prime} \in\{\mathcal{B} \backslash m\} .
\end{aligned}
$$

Finally, we evaluate the hourly KPI metric for BS $m$ at time $t, K P I_{m}(t)$, that is defined as the ratio between the total data volume (DV) transmitted and the energy consumed (E) during one hour.

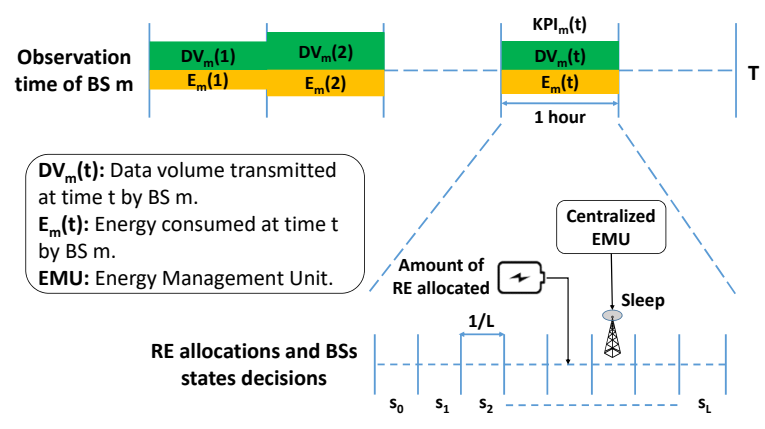

Fig. 1: Dynamic network operations.

\section{Energy Consumption Model}

In order to calculate the power consumption of the wireless cellular network, we use the power model provided by EARTH [16]. Accordingly, the power consumption of a BS consists of a static part $P_{0}$ and a traffic dependent part $P_{\text {out }}$. Moreover the power demand depends on the number of active RBs. Because of the nearly linear relationship that exists between the RF power and the BS power consumption, the power consumed by a BS at time slot $i$ can be expressed as follows:

$$
\begin{aligned}
& P_{\text {in }, i}= \\
& \begin{cases}N_{\text {trx }}\left(P_{0}+\frac{n_{R B}^{(i)}}{n_{R B}^{\text {Total }}} \times \Delta_{p} \times P_{\text {out }}^{(i)}\right), & 0<P_{\text {out }} \leq P_{\text {max }}, \\
N_{\text {trx }} P_{\text {sleep }}, & P_{\text {out }}=0 .\end{cases}
\end{aligned}
$$

where $N_{t r x}$ is the number of transceivers. $\Delta_{p}$ and $P_{\text {sleep }}$ are the load dependent parameter and the power con- 
sumption of the BS in sleep mode, respectively. $n_{R B}^{(i)}$ and $n_{R B}^{T o t a l}$ are the number of active RBs at time slot $i$ and the maximum number of RBs available to the BS, respectively.

\section{Downlink Transmission Model}

We measure the downlink transmission quality between a BS $m$ and a user $u$ based on the SINR as follows:

$$
\operatorname{SINR}_{m}(u)=\frac{P_{m} h_{m}(u)}{\sigma^{2}+\sum_{m^{\prime} \in \mathcal{B}, m^{\prime} \neq m} P_{m^{\prime}} h_{m^{\prime}}(u)}
$$

where $P_{m}$ is the transmitted power of BS $m, h_{m}(u)$ is the channel gain from BS $m$ to user $u$, which accounts for the path loss and shadowing effect, and $\sigma^{2}$ is the additive white Gaussian noise power density.

We can express the rate offered to a user $u$ and served by BS $m$ using Shannon-Hartley theorem as follows:

$$
R_{m}(u)=n_{R B}(u) \times \mathrm{BW}_{\mathrm{RB}} \times \log _{2}\left(1+\operatorname{SINR}_{m}(u)\right)
$$

where $n_{R B}(u)$ is the number of RBs allocated to the user $u$, and $\mathrm{BW}_{\mathrm{RB}}$ is the bandwidth of one RB.

\section{E. Renewable Energy Generation and Traffic Variation}

We consider the use of solar panels due to their flexibility. In addition, we provide these sites with battery storages in order to store the excess harvested energy for future transmission. The amount of harvested energy varies depending on the location and panel size. In Fig. 2 , we illustrate an example of the harvested energy generated by a solar panel having a surface area of $12.5 \mathrm{~m}^{2}$ in the city of Marseille (France) [17]

The traffic load on the other hand manifests both temporal and spatial diversities. On the temporal diversity, the traffic load of a BS dynamically changes over time as shown in Fig. 2. We assume that the users are uniformly distributed in the given area.

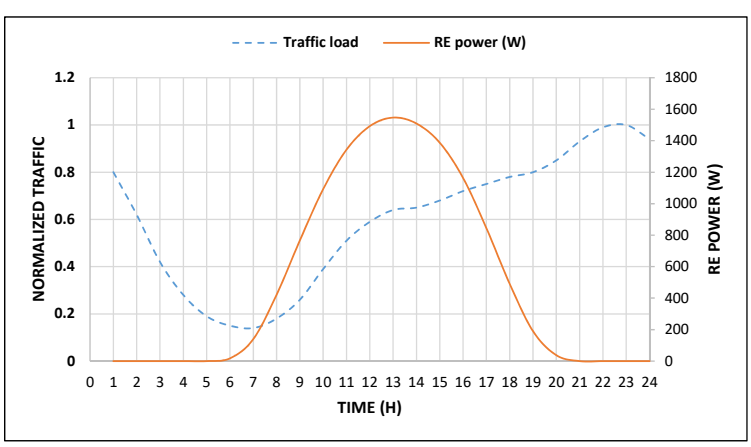

Fig. 2: Variations of the average traffic load and the average RE power [16], [17].

III. ADVANCED

\section{ENERGY KPIS AND PROBLEM FORMULATION}

The focus in our work is on EE-KPIs for wireless communications. Therefore, the bits per Joule metric understudy is given as follows:

$$
K P I=\frac{D V}{E}(\mathrm{bit} / \mathrm{J})
$$

where $D V$ represents the overall volume of transmitted data in bits and $E$ is the consumed energy in Joules. Let us first define the following new EE-KPIs that take into consideration the usage of RE (in the rest of the paper, we often refer to EE-KPI as KPI for the sake of abbreviation):

Definition 1 (Global KPI): We denote by global KPI, the hourly performance evaluation of the EE of a BS including all the services it is providing. It is calculated as follows:

$$
K P I_{m}(t)=\frac{D V_{m}(t)}{E_{m}(t)-R E_{m}(t)}
$$

where $R E_{m}(t)$ is the allocated RE for BS $m$ at time $t$, and it depends on the energy harvesting policy.

Definition 2 (Service KPI): The service $i$ KPI is the hourly EE evaluation of service $i$ provided by BS $m$ as follows:

$$
K P I_{m}^{i}(t)=\frac{D V_{m}^{i}(t)}{E_{m}^{i}(t)-R E_{m}^{i}(t)}
$$

where $D V_{m}^{i}$ is the volume of the transmitted data by service $i$, and $E_{m}^{i}$ is the energy consumed by this service. The latter is evaluated based on Shapley model presented in [13] and is summarized in the next subsection.

We now extend the above mentioned KPIs by defining a new metric scale under which we evaluate our EE-KPIs: Network KPI. This metric can be used to evaluate either global or services KPIs.

Definition 3 (Network KPI): The network KPI, $\operatorname{KPI}\left(t_{1}, t_{2}\right)$ is defined as the hourly average EE of the entire network including BSs in sleep mode, between times $t_{1}$ and $t_{2}$, where $\mathbf{K P I}\left(t_{1}, t_{2}\right) \triangleq[K P I(t)]_{t_{1} \leq t \leq t_{2}}$ and:

$$
K P I(t)=\frac{1}{M} \sum_{m=1}^{M} \frac{D V_{m}(t)}{E_{m}(t)-R E_{m}(t)}
$$

Since this metric is evaluated hourly, it takes hourly discrete values. For example, $\mathbf{K P I}(1,24)=[K P I(1), K P I(2), \ldots, K P I(24)]$, where $K P I(1)$ is evaluated at time $=1$ hour.

\section{A. Shapley Model for Energy Sharing among Services}

While it is easy to monitor the traffic volume consumed by service $i$, its specific energy consumption, $E_{i}$, is not intuitive. In order to evaluate the energy consumed by each service, we use the model proposed in [13] that estimates this energy based on Shapley Value, a coalition game concept.

The energy consumption of the network is composed of two components: a variable one $\left(E^{v}\right)$ that varies with the traffic load and a fixed one $\left(E^{f}\right)$ independent of the network load. These two components can be easily monitored using appropriate energy consumption models such as EARTH model (summarized in Section II-C). Similarly, we 
can evaluate the energy consumed by service $i$ as follows:

$$
E_{i}=E_{i}^{f}+E_{i}^{v}
$$

While the variable part is shared among the services with proportion to the service traffic, i.e., $E_{i}^{v}=D V_{i} / D V_{T} \times E^{v}\left(D V_{T}\right.$ is the total data volume), the fixed part is estimated following Shapley Value as follows:

$$
\begin{aligned}
& E_{i}^{f}=\theta_{i} \times E^{f} \theta_{i}\left(N, p_{i}\right)= \\
&\left(\sum_{s=1}^{N} \frac{1}{s C_{N}^{s}}\right) p_{i} \\
&+\left(\sum_{s=2}^{N} \frac{\left(C_{N-1}^{s-2}-C_{N-1}^{s-1}\right) C_{N-2}^{s-2}}{C_{N-1}^{s-1} C_{N-1}^{s-2} s C_{N}^{s}}\right)\left(1-p_{i}\right)
\end{aligned}
$$

where $\theta_{i}$ is the share of energy for service $i$ following Shapley Value, $N$ is the number of services (players), $p_{i}$ is the traffic portion $\left(p_{i}=\frac{D V_{i}}{D V_{T}}\right)$, and $C_{N}^{s}$ is the binomial coefficient.

Compared to uniform and proportional sharing of fixed energy component, Shapley model achieves a trade-off among all services. That is to say, it does not penalize major services as much as proportional sharing, and it is also a good trade-off for small services as it does not condemn them with high fixed energy as in uniform sharing. By combining (7), (9) and (10), the EE-KPI of service $i$ is evaluated as follows:

$$
K P I_{m}^{i}(t)=\frac{D V_{m}^{i}(t)}{\theta_{i} \times E_{m}^{f}(t)+\phi_{i} \times E_{m}^{v}(t)-R E_{m}(t)}
$$

where $\phi_{i}$ is the proportional share of the service variable energy, $\phi_{i}=D V_{i} / D V_{T} . E_{m}^{f}$ and $E_{m}^{v}$ are calculated using EARTH model in Section II-C.

\section{B. Problem Formulation}

Considering both QoS and battery capacity constraints, we aim at maximizing the EE-KPI by the efficient use of RE. We consider a network of LTE BSs having a percentage of its sites equipped with RE. We assume that the BSs always have data to transmit. Our goal is to maximize the average network KPI over a time duration $\mathrm{T}$. We express the optimization problem as follows:

$$
\max _{\mathbf{R E}(T), \mathbf{S}(T)} \frac{1}{T} \sum_{t=t_{1}}^{t_{2}} K P I(t)
$$

subject to:

Quality constraint:

$$
r(u) \geq R_{\text {min }}, \forall u \in \mathcal{U}_{m}, \forall m \in \mathcal{B}
$$

System constraint:

$$
\begin{gathered}
\frac{\sum_{u \in \mathcal{U}_{m}} w_{u}}{W} \leq 1, \forall m \in \mathcal{B} \\
P_{m} \leq P_{\max }
\end{gathered}
$$

Renewable energy availability constraint:

$$
R E_{m}(t) \leq B_{m}(t)
$$

where $w_{u}$ is the bandwidth allocated for user $u$. RE and $\mathbf{S}$ are the RE utilization for each BS equipped with $\mathrm{RE}$ and the state of each BS (active or sleep) in the network at each time horizon, respectively. $R E_{m}(t)$ and $B_{m}(t)$ are the allocated harvested energy and the available energy stored in the battery for BS $m$, at the beginning of stage time $t$, respectively; where $R E_{m}^{s}(t)=R E_{m}^{s}(t-1)+R E_{m}^{a}(t)-R E_{m}(t)$, and $R E_{m}^{s}(t)$ and $R E_{m}^{a}(t)$ are the stored energy and the harvested energy allocated by BS $m$ at time $t$, respectively.

Problem (13) is a non-convex fractional problem due to its objective function. In the next section, we propose a heuristic online algorithm that efficiently utilizes the harvested energy, and decides which BSs to switch to sleep state in order to improve the EE-KPI. The above formulated problem maximizes the network global KPI. The same problem can be formulated for the services KPIs.

\section{IV.}

\section{AdAPtATION OF ENERGy MANAGEMENT STRATEGIES}

Enhancing the EE-KPI requires maximizing the throughput of the network while minimizing the on-grid energy consumption. The two parameters that directly affect this improvement are traffic load and RE. SPAEMA, an energy management algorithm described in [9], is an online algorithm that decides how to manage RE (store it in battery or use it) based on the battery state of charge (SoC) and the price of electricity, in order to reduce the electric bill of the operator. Thus, it does not require any future knowledge of RE nor traffic load. In [9], we showed that SPAEMA outperforms the traditional algorithm that uses $\mathrm{RE}$ whenever it is available. However, since the objective has shifted from reducing the electric bill of the operator to increasing our EE-KPIs, adaptation of our previously proposed algorithm (SPAEMA) is needed to match our new objective. Thus, we propose a new algorithm that adapts SPAEMA to satisfy the newly introduced objective. Then we extend the proposed algorithm for further enhancement.

\section{A. Proposed algorithm 1 - STAEMA}

We suggest a Simplified Traffic-Aware Energy Management Algorithm (STAEMA) that is an adaptation of SPAEMA. The major motivation behind this adaptation is to preserve the simplicity of SPAEMA and avoid high complexity. In contrast to SPAEMA that ignores the traffic load of the network, which is an important parameter in our studied KPIs, our proposed algorithm manages the use of RE by taking into account the SoC of the battery and the traffic load. Similar to SPAEMA, we quantify each of the traffic and battery storage states into 3 discrete levels: low, medium and high. Table I shows the applied decisions for different cases.

The decisions summarized in the table below are designed to store the harvested energy in the battery when its SoC is low, and then use it at high traffic load. This 
TABLE I: STAEMA decisions for different cases of battery and traffic load.

\begin{tabular}{|c|c|c|c|}
\hline Battery/Traffic & Low & Medium & High \\
\hline \hline Low & store & store & use \\
\hline Medium & store & use & use \\
\hline High & use & use & use \\
\hline
\end{tabular}

setup will minimize the waste of RE caused by battery overflow, and compensate for the increase in energy consumption caused by the increased traffic load.

Both SPAEMA and STAEMA don't allow BSs $\in B S^{m i x}$ to switch to sleep mode. In the next algorithm, we extend the latter algorithm to allow hybrid powered BSs to switch to sleep mode.

\section{B. Proposed algorithm 2 - Extended-STAEMA}

Extended-STAEMA (Ext-STAEMA) allows BSs equipped with RE sources to switch to sleep mode. It modifies the candidate set $\mathcal{B}^{c}$ to include $\mathrm{BSs} \in B S^{\text {mix }}$.

When a BS switches to sleep mode, its EE-KPI drops to zero, since it is not transmitting data anymore, and hence, affecting the network EE-KPI as shown in Fig. 3. At $t_{1}$, both BSs are active (consuming energy and transmitting data) with different KPI values. At $t_{2}$, $B S_{2}$ switches to sleep mode. During this time, the users offload to $B S_{1}$, increasing its data transmitted volume, thus increasing its KPI, while the KPI of $B S_{2}$ drops to zero. In order to better handle this degradation in the network EE-KPI, we decide to put the BSs equipped with RE sources to sleep, in the case where the traffic load is high and the battery SoC is low. The motivation behind this is to preserve the energy stored in the battery for a longer period of time and to enhance the KPI of the neighboring BSs by transmitting more data to the newly offloaded users. We summarize the decisions in Table II.

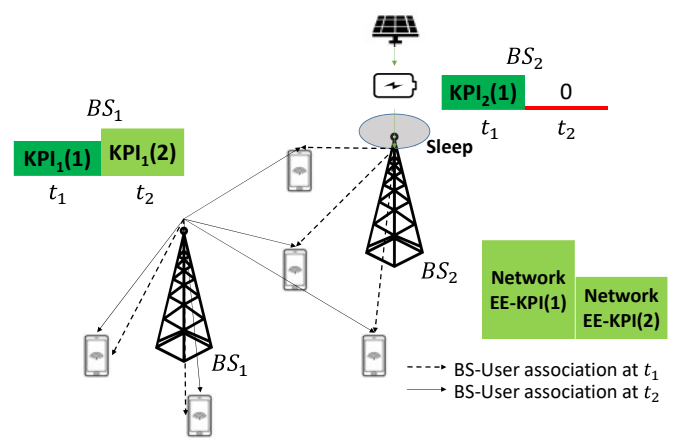

Fig. 3: Illustration of the problem: At $t_{1}$, both BSs are active. At $t_{2}, B S_{2}$ switches to sleep mode, and its users offload to $B S_{1}$.

The decision of switching BS $m \in B S^{m i x}$ to sleep mode, in the case when the SoC of its battery is low and the traffic is high, depends whether the condition of switching off $m$ is satisfied (Eq.(1)). If not, $m$ will use its stored energy to power its components.
TABLE II: Extended-STAEMA decisions for different cases of battery and traffic load.

\begin{tabular}{|c|c|c|c|}
\hline Battery/Traffic & Low & Medium & High \\
\hline \hline Low & store & store & use/ sleep \\
\hline Medium & store & use & use \\
\hline High & use & use & use \\
\hline
\end{tabular}

\section{Simulation AND Results}

In this section, we provide simulation results to assess the network performance by evaluating the EE-KPIs of the services provided by a cellular network under the proposed algorithms. We obtain our results via Monte-Carlo method using MATLAB. The month of June is considered as an example to harvest RE, due to its high solar potential $\left(7.08 K W h / d a y / m^{2}\right)$ [17]. We assume that the load is randomly distributed among the BSs following the traffic load variation in Fig. 2. We further consider five service categories: two large ones (streaming and web) and three smaller ones (download, voice and other minor data services). We set $T=24$ hours and evaluate the $K P I(t)$ on an hourly basis. From Eq. (6), whenever the harvested energy $\left(R E_{m}(t)\right)$ is higher than the energy consumed by BS $m\left(E_{m}(t)\right)$, the excess $\mathrm{RE}$ is stored in the battery. In table III, we summarize the simulation parameters and traffic proportions of each service taken from a real European operator data set [13].

TABLE III: Parameters' values and assumptions.

\begin{tabular}{|c|c|}
\hline Parameters & Values \\
\hline Number of BSs & 25 \\
\hline Number of sectors & 3 \\
\hline Bandwidth & $10 \mathrm{MHz}, \mathrm{FDD}$ \\
\hline Maximum transmitted power & $43 \mathrm{dBm}$ \\
\hline Inter-cell distance & $1000 \mathrm{~m}$ \\
\hline RB & 50 \\
\hline BW $\mathrm{RB}$ & $180 \mathrm{KHz}$ \\
\hline Number of users K & 400 \\
\hline User min. required rate & $1 \mathrm{Mb} / \mathrm{s}$ \\
\hline Noise power & $-174 \mathrm{dBm} / \mathrm{Hz}$ \\
\hline$P_{0}$ & $118.7 \mathrm{~W}$ \\
\hline$\Delta_{p}$ & 5.32 \\
\hline Number & 10 \\
\hline of slots per hour period L & \\
\hline Battery capacity & $5 \mathrm{KWh}$ \\
\hline Services & Traffic proportions \\
\hline Streaming & $34 \%$ \\
\hline Web & $30 \%$ \\
\hline Download & $13 \%$ \\
\hline Voice & $9 \%$ \\
\hline Other data & $14 \%$ \\
\hline
\end{tabular}

\section{A. Impact or RE and sleep scheme on EE-KPI}

In Fig. 4, we evaluate the network EE-KPI for the different services the network is providing. These 
values are calculated in the absence of RE and energy management strategies. From the obtained results, we observe that the EE-KPIs of the services are proportional to their traffic volumes, i.e., the larger the service is, the higher is its KPI. And even though Shapley energy model puts more weight on larger service categories, their EE-KPI is higher due to their increased traffic volume.

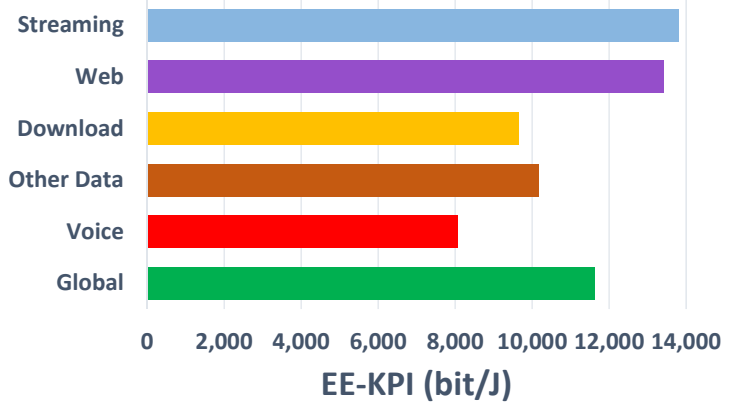

Fig. 4: Evaluation of the network global and services EE-KPI in the absence of RE and energy management techniques.

In Fig. 5, we evaluate the impact of RE and sleep scheme under the Extended-STAEMA on the streaming service network EE-KPI. We will focus on streaming service due to its high impact on the KPI as shown in Fig. 4.

Using solar panels that only harvest $35 \%$ of the total energy demand at full load, we are able to enhance the EE-KPI by about a factor of 10 when $25 \%$ of the sites are equipped with RE and by roughly 40-times when all the BSs of the network are equipped with RE. This significant increase in the KPI is demonstrated in Eq. (8). From this equation, this metric can go up to very large values when significant amount of RE is available, i.e., when this amount is equal to the energy required by the BS. However, and in order to handle these massive EE-KPIs, we set them to high values compared to the average network KPI. On the other hand, when sleep scheme is applied we observe a slight decrease in the network EE-KPI $(\sim 9 \%)$. The reason behind this is twofold. First, the network EE-KPI takes into account operational and inactive (sleep) BSs having positive and zero KPIs, respectively. So the more inactive BSs we have, the less the network EE-KPI becomes. The second reason occurs when a BS goes to sleep mode and some of its users offload to another BS equipped with RE source. In this case, both the energy demand and the data volume transmitted by the latter BS will increase. However, if this happens at the end of the day, when the battery storage is suffering due to low RE, this increase in energy demand might force the BS to draw energy from the grid in order to compensate for this surge in energy consumption. This will result in a significant drop in its KPI, especially since it was solely relying on its green stored energy. We will show next that despite this inconvenience, some energy savings can be attained with sleep strategy.

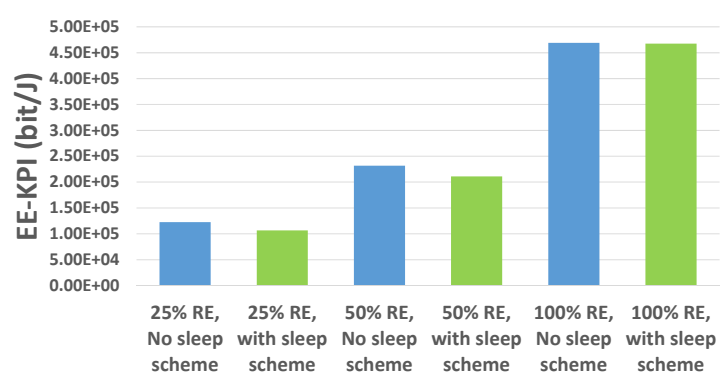

Fig. 5: Network streaming EE-KPI with Ext-STAEMA.

\section{B. Adaption of energy management techniques}

We use greedy algorithm and SPAEMA as benchmarks to compare with our proposed algorithms. In greedy algorithm, the BS uses the harvested energy whenever available and stores the excess RE in the battery.

Fig. 6 illustrates the performance of the network streaming EE-KPI for different percentages of sites equipped with RE without sleep mode. The system achieves the lowest EEKPI when the energy allocation policy does not take into account traffic volume (greedy and SPAEMA). On the other hand, STAEMA results in an enhancement in the EE-KPI up to $11.45 \%$ in comparison with the other two. The reason behind the similar performances of greedy and SPAEMA algorithms is that they both ignore traffic load variations.

In Fig. 7, we present the results in the case of sleep scheme. Ext-STAEMA algorithm, that widens the candidate set of BSs allowed to switch to sleep mode to include BSs equipped with RE, performs slightly better than STAEMA $(\sim 2 \%)$. This tells us that under these traffic-aware algorithms, our EE-KPI has reached its limit. The reason is because our metric does not valorize grid energy savings of the network. Despite having similar performances of EE-KPI, Ext-STAEMA saves more energy than the other algorithms (up to $8 \%$ more savings), as shown in Table IV. This can be explained by the wider set of BSs that can be put to sleep mode, and thus saving more energy.

TABLE IV: Total energy savings of the network.

\begin{tabular}{|c|c|c|c|c|}
\hline \multirow{2}{*}{ Algorithms } & \multicolumn{4}{|c|}{ Total Energy Savings (\%) } \\
\cline { 2 - 5 } & $\%$ of sites equipped with RE \\
\cline { 2 - 5 } & $25 \%$ & $50 \%$ & $75 \%$ & $100 \%$ \\
\hline Greedy & 37.75 & 40.8 & 44.3 & 50.5 \\
\hline SPAEMA & 37.75 & 40.8 & 44.3 & 50.5 \\
\hline STAEMA & 38.13 & 40.8 & 44.3 & 50.5 \\
\hline Ext-STAEMA & 40.46 & 45.5 & 51.7 & 58.4 \\
\hline
\end{tabular}

\section{CONCLUSION}

In this paper, we have studied advanced services-based energy KPIs. These metrics are important for the evaluation of future cellular networks by putting forward the contribution of each service to energy consumption. Our research focuses on the adaptation of energy 


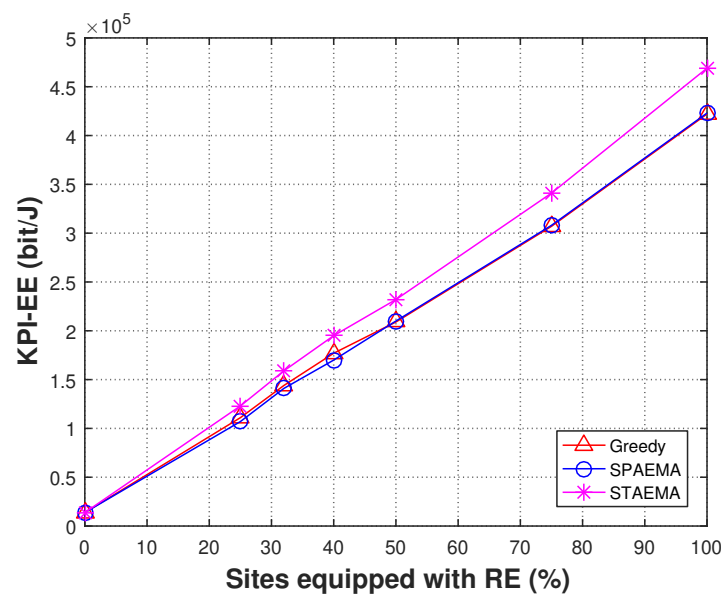

Fig. 6: Network streaming EE-KPI without sleep scheme.

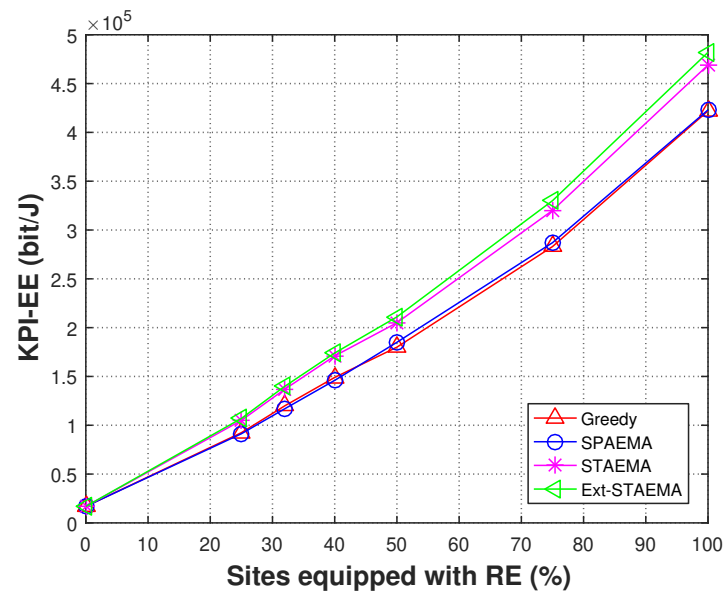

Fig. 7: Network streaming EE-KPI with sleep scheme.

management strategies with the objective of having better energy indicators for each of the services.

The context studied allows to investigate different parameter choices. For instance, we can study the impact of the type of batteries used, their capacities and their ageing calender on the performance of cellular networks in terms of EE.

\section{ACKNOWLEDGMENTS}

The authors would like to acknowledge the support of the French Ministry of Industry for supporting this work under SooGREEN project.

\section{REFERENCES}

[1] W. V. Heddeghem, S. Lambert, B. Lannoo, D. Colle, M. Pickavet, and P. Demeester, "Trends in worldwide ICT electricity consumption from 2007 to 2012," Computer Communications, vol. 50, no. Supplement C, pp. 64 - 76, 2014. Green Networking

[2] E. Gelenbe and Y. Caseau, "The impact of information technology on energy consumption and carbon emissions," pp. 1-15, 062015

[3] H. Holtkamp, G. Auer, S. Bazzi, and H. Haas, "Minimizing base station power consumption," IEEE Journal on Selected Areas in Communications, vol. 32, pp. 297-306, February 2014.
[4] H. Kwon and T. Birdsall, "Channel capacity in bits per joule," IEEE Journal of Oceanic Engineering, vol. 11, pp. 97-99, January 1986.

[5] J. Wu, Y. Zhang, M. Zukerman, and E. K. N. Yung, "Energyefficient base-stations sleep-mode techniques in green cellular networks: A survey," IEEE Communications Surveys Tutorials, vol. 17, pp. 803-826, Secondquarter 2015.

[6] F. Han, S. Zhao, L. Zhang, and J. Wu, "Survey of strategies for switching off base stations in heterogeneous networks for greener 5G systems," IEEE Access, vol. 4, pp. 4959-4973, 2016.

[7] H. ElSawy, E. Hossain, and M. Haenggi, "Stochastic geometry for modeling, analysis, and design of multi-tier and cognitive cellular wireless networks: A survey," IEEE Communications Surveys Tutorials, vol. 15, pp. 996-1019, Third 2013.

[8] H. A. H. Hassan, L. Nuaymi, and A. Pelov, "Renewable energy in cellular networks: A survey," in IEEE Online Conference on Green Communications (OnlineGreenComm), pp. 1-7, Oct 2013

[9] H. A. H. Hassan, L. Nuaymi, and A. Pelov, "Classification of renewable energy scenarios and objectives for cellular networks," in IEEE 24th Annual International Symposium on Personal, Indoor, and Mobile Radio Communications (PIMRC), pp. 2967-2972, Sept 2013.

[10] C. Liu, B. Natarajan, and H. Xia, "Small cell base station sleep strategies for energy efficiency," IEEE Transactions on Vehicular Technology, vol. 65, pp. 1652-1661, March 2016.

[11] R. Ramamonjison and V. K. Bhargava, "Energy allocation and cooperation for energy-efficient wireless two-tier networks," IEEE Transactions on Wireless Communications, vol. 15, pp. 6434-6448, Sept 2016.

[12] J. Hu, G. Zhang, W. Heng, and X. Li, "Optimal energy-efficient transmission in multiuser systems with hybrid energy harvesting transmitter," in 2016 IEEE Global Communications Conference (GLOBECOM), pp. 1-5, Dec 2016.

[13] W. Yoro, T. Chahed, M. E. Tabach, T. En-Najjary, and A. Gati, "Sharing of energy among service categories in wireless access networks using shapley value," in 2016 International Wireless Communications and Mobile Computing Conference (IWCMC), pp. 422-429, Sept 2016.

[14] ETSI, "Measurement method for power consumption and energy efficiency of wireless access network equipment, ES 202 706," 2014.

[15] A. E. Amine, H. A. H. Hassan, and L. Nuaymi, "Analysis of energy and cost savings in hybrid base stations power configurations," in IEEE Vehicular Technology Conference (VTC Spring), 2018.

[16] G. Auer, V. Giannini, C. Desset, I. Godor, P. Skillermark, M. Olsson, M. A. Imran, D. Sabella, M. J. Gonzalez, O. Blume, and A. Fehske, "How much energy is needed to run a wireless network?," IEEE Wireless Communications, vol. 18, pp. 40-49, October 2011.

[17] "PVWatts calculator." last checked: February 2018. 